\title{
Transformation d'un restaurant hospitalier en unité de soins critiques de courte durée
}

\author{
Transformation of a Hospital Restaurant into an Orientation Intensive Care Unit
}

\author{
M. Borel · P. Lhermite $\cdot$ N. Fleury $\cdot$ M.-A. Ruder $\cdot$ C. Fazilleau $\cdot$ E. Boudon $\cdot$ T. Lemarec $\cdot$ J. Mayaux \\ J.-M. Constantin · M. Raux
}

Reçu le 28 juin 2021 ; accepté le 12 juillet 2021

(C) SFMU et Lavoisier SAS 2021

Résumé En mars 2021, l'île-de-France a connu une nouvelle vague de Covid-19. Face à une projection épidémique faisant craindre un dépassement des capacités, la cellule de crise de l'Assistance publique-Hôpitaux de Paris a validé la mise en œuvre du projet Météor. Météor est la transformation d'un restaurant du personnel du CHU Pitié-Salpêtrière en réanimation d'accueil et d'orientation de patients Covid19 avant qu'ils ne soient adressés à des réanimations plus pérennes sur site ou en dehors. Cette démarche n'ayant jamais été pensée auparavant, tout était à construire. Une équipe projet composée d'un cadre de santé, d'un médecin, d'un ingénieur et d'un directeur a piloté sa mise en œuvre. Le projet s'est voulu modulable, démontable et intégré comme une ressource zonale. En à peine 15 jours, l'unité était opérationnelle. L'organisation des soins a été simplifiée et standardisée au maximum pour que chaque personnel

M. Borel $(\bowtie) \cdot$ N. Fleury $\cdot$ C. Fazilleau $\cdot$ J.-M. Constantin ·

M. Raux

Département médico-universitaire réanimation-anesthésie

médecine périopératoire (DREAM),

groupe hospitalier Pitié-Salpêtrière-Charles-Foix,

Assistance publique-Hôpitaux de Paris,

Sorbonne Université (AP-HP SU),

F-75651 Paris cedex 13, France

e-mail : marie.borel2@aphp.fr

P. Lhermite

Direction des investissements et des systèmes numériques, groupe hospitalier Pitié-Salpêtrière-Charles-Foix, AP-HP SU, 47-83, boulevard de l'Hôpital,

F-75651 Paris cedex 13, France

M.-A. Ruder

Direction, groupe hospitalier Pitié-Salpêtrière-Charles-Foix, AP-HP SU, 47-83, boulevard de l'Hôpital,

F-75651 Paris cedex 13, France

E. Boudon

Direction des équipements biomédicaux et mobiliers,

groupe hospitalier Pitié-Salpêtrière-Charles-Foix, puisse assurer sa mission en sérénité malgré un contexte et des lieux inhabituels. L'efficacité de la mise en œuvre du projet est liée à l'expertise des équipes en place et au caractère multiprofessionnel de l'équipe projet. Le point de fragilité s'est avéré être les ressources humaines. La constitution d'un corps de réserve sanitaire de personnels de soins critiques est nécessaire pour anticiper la prochaine crise.

Mots clés Covid-19 - Gestion de crise · Centre médical de tri et d'évacuation

Abstract In March 2021, the Île-de-France area was experiencing a new wave of Covid-19. Faced with an epidemic projection that could exceed capacity, the Assistance publique-Hôpitaux de Paris (AP-HP) crisis unit validated the implementation of the Meteor project. The Meteor is

AP-HP SU, 47-83, boulevard de l'Hôpital,

F-75651 Paris cedex 13, France

T. Lemarec

Pharmacie à usage intérieur,

groupe hospitalier Pitié-Salpêtrière-Charles-Foix, AP-HP SU, 47-83, boulevard de l'Hôpital, F-75651 Paris cedex 13, France

\section{J. Mayaux}

Département médico-universitaire assistance respiratoire, addictologie, tabacologie, pneumologie, physiologie, réhabilitation, réanimation, oncologie, chirurgie thoracique, handicap, endoscopie, sommeil (APPROCHES), groupe hospitalier Pitié-Salpêtrière-Charles-Foix, AP-HP SU, 47-83, boulevard de l'Hôpital,

F-75651 Paris cedex 13, France

J.-M. Constantin · M. Raux

UMRS Inserm 1158, Sorbonne Université, F-75013 Paris, France 
the transformation of a restaurant within the Pitié-Salpêtrière University Hospital into a Covid-19 patient orientation intensive care unit before they are sent to more permanent ICUs on or off site. As this approach had never been thought of before, everything had to be constructed. A project team composed of a chief nurse, a physician, an engineer and a director piloted its implementation. The project was designed to be modular, dismantleable and integrated as a zonal resource. In barely 15 days, the unit was operational. The organization of care was simplified and standardized to the maximum so that each staff member could carry out his or her mission with serenity despite the unusual context and locations. The effectiveness of project implementation is linked to the expertise of the teams in place and the multiprofessional nature of the project team. The weakest point was human resources. The constitution of a health reserve body of critical care personnel is necessary to anticipate the next crisis.

Keywords Covid-19 · Crisis management · Medical sorting $\cdot$ Evacuation center

\section{Introduction}

Fin mars 2021, l'Île-de-France faisait face de nouveau à une augmentation importante du nombre de patients Covid-19 nécessitant le recours aux soins critiques. Les hôpitaux de l'Assistance publique-Hôpitaux de Paris (AP-HP) se sont mobilisés pour augmenter le nombre de lits de soins critiques. Les projections épidémiologiques étaient alarmistes [1] et faisaient craindre que ces efforts soient insuffisants pour passer le pic de la crise en cours. La cellule de crise de l'AP-HP et celles du groupe hospitalier ont une nouvelle fois confrontées à la nécessité pour faire face de sortir du cadre et penser autrement [2]. La crise déstructurante a dû être acceptée et analysée pour pouvoir piloter dans une situation hautement incertaine [3]. Dans ce contexte, le projet Météor a été initié et soutenu par la direction de l'AP-HP, piloté par la cellule de crise du groupe hospitalier AP-HP-Sorbonne Université (AP-HP-SU) construit en interaction avec le Samu zonal. Les lignes à suivre en sont le retour d'expérience.

\section{Projet}

L'objectif de Météor était de permettre à des patients qui ont des critères d'éligibilité aux soins critiques, mais qui ne pouvaient y être admis faute de place disponible en Îlede-France, dans un court délai de bénéficier au plus vite de soins de réanimation. Il s'agissait de créer un lieu supplémentaire, en dehors des sites de réanimation existants (car surchargés), capable d'accueillir en tout temps un patient nécessitant des soins critiques du fait de la Covid-19. Ce projet a été construit comme modulable, permettant une adaptation en fonction du besoin : zone de soins et de transit avant accès à la réanimation, unité de soins critiques en cas de débordement des capacités disponibles, unité de postréanimation permettant de libérer des places en réanimation, zone d'accueil d'une réanimation en cas d'incapacité (incendie, panne électrique, inondation...) à vocation régionale, voire extrarégionale. Météor s'est inscrit dans la remarquable réactivité de l'hôpital public devant une crise nouvelle par son intensité et sa durée.

Le critère d'admission était la nécessité de délivrer des soins critiques à un patient fortement suspect ou confirmé de Covid-19 et un délai de régulation supérieur à une heure. L'objectif était ainsi de poursuivre une prise en charge sécurisée en termes de qualité de soins tout en construisant le parcours du patient et son orientation. Le patient était ainsi admis en unité d'accueil où le diagnostic Covid était confirmé et les premiers éléments de prise en charge cliniques, techniques et administratifs réalisés. En l'absence de confirmation diagnostique de Covid-19, le patient aurait été extrait de la zone et réorienté en fonction des ressources disponibles dans l'établissement de santé ou ailleurs. En cas de confirmation, le patient aurait été orienté au sein de la zone de soins dédiée du projet Météor. Dès l'arrivée du patient au sein de la structure, une démarche de recherche de place pérenne aurait été initiée par le Samu zonal, au besoin en lien avec l'ARS et le centre de crise sanitaire. Le projet Météor était celui d'une unité de réanimation d'accueil et d'orientation de patients Covid en situation pandémique avec un dépassement du capacitaire. Il s'agissait donc d'un contexte exceptionnel de médecine de catastrophe. En dépit de ce contexte exceptionnel, la volonté devait être de garder un niveau élevé de qualité et de sécurité des soins. Les patients n'avaient pas vocation à être admis au sein de Météor si des places de soins critiques étaient disponibles ailleurs. De même, les patients n'avaient pas vocation à y rester dès lors que des places se libéraient et qu'ils étaient éligibles à la poursuite de la réanimation. En l'absence d'éligibilité à la réanimation, les thérapeutiques engagées en préhospitalier pouvaient être limitées ou interrompues au sein d'une zone de soins dédiée à l'accompagnement de fin de vie. Les modélisations anticipant un dépassement des capacités d'hospitalisation, cette unité a été construite pour permettre malgré tout si nécessaire une prise en charge prolongée. S'inscrivant délibérément dans une logique de flux de patients et non de lits statiques, Météor avait pour objectif de toujours offrir des places de réanimation au Samu ou au service d'urgence dans le but de prévenir des morts évitables autant que de mobiliser indûment des équipes préhospitalières soumises à forte tension. Météor a conduit à repenser le parcours de soins critiques en situation de moyens dépassés : le parcours de réanimation d'un patient ne se fait plus en un seul et même service. 


\section{Modalités de construction du projet}

Une fois définis les grands axes de travail pour l'élaboration du projet Météor, le projet s'est mis en route de façon collaborative et multiprofessionnelle en associant un médecin, un cadre, un ingénieur et un directeur. Cette cellule de pilotage opérationnel partant d'une feuille blanche devait aller vite (Fig. 1). Chacun a mené son axe de travail en parfaite collaboration et en toute confiance avec les trois autres [4]. Le projet de soins a été construit en interaction avec le projet architectural. Il fallait de concert construire le projet médical, organiser les soins, choisir et trouver les équipements, et résoudre l'ensemble des difficultés techniques et de construction. Le projet a par ailleurs été présenté aux organisations syndicales, membres du comité d'hygiène et de sécurité des conditions de travail, en plus des différentes discussions en cellules de crise afin qu'il soit, malgré sa nature inhabituelle, conforme aux obligations légales, compris et accepté par l'ensemble de la communauté hospitalière.

\section{Choix du site}

Le choix du CHU Pitié-Salpêtrière était adapté aux objectifs dans la mesure où l'hôpital est à un carrefour de communication : ferroviaire (gare d'Austerlitz à partir de laquelle des trains " chardons » sont partis pour la première vague), aérien (aéroport d'Orly à partir duquel des patients sont partis en transferts interrégionaux), hélistation et autoroutiers $[5,6]$. Le choix de cet établissement était aussi pertinent dans la mesure où les circuits logistiques, techniques, pharmaceutiques, biomédicaux sont déjà présents dans l'hôpital ainsi que l'expertise technique comme soignante pour mener pareille démarche. Ce choix permettait de ne pas avoir à recréer l'ensemble de ces circuits d'expertise mais d'ajouter un site supplémentaire avec une fonctionnalité définie et différente en complément de l'existant. Par ailleurs, le CHU Pitié-Salpêtrière avait déjà l'expertise multiprofessionnelle de la création d'unités éphémères de soins critiques (comme par exemple la transformation du bâtiment de chirurgie ambulatoire en réanimation lors de la première vague en mars 2020) avec toutefois une différence pour ce projet qui était de construire dans un lieu habituellement non dédié aux soins. Pour faire face à la nécessité de franchir un palier dans l'augmentation de la capacité d'accueil de soins critiques, avec une typologie d'activité modulable en fonction des besoins, la zone choisie devait être de grande taille, non déjà occupé par une activité de soins, et pouvant être reliée à l'ensemble des fluides (oxygène, air comprimé médical, vide) et réseaux nécessaires (électricité, téléphonie, internet) à une unité de réanimation. Le lieu devait par ailleurs être facile d'accès pour les ambulances. Le choix d'un des deux restaurants hospitaliers a été fondé sur sa localisation au sein du site, à la fois proche d'un bâtiment de réanimation, de la pneumologie, mais également proche du bâtiment de laboratoires. Le restaurant hospitalier présente l'avantage d'être un espace d'environ $700 \mathrm{~m}^{2}$, d'un volume important intégrant des réseaux de traitement d'air. Ces caractéristiques, et le fait qu'il soit alimenté et relié aux autres constructions via des galeries techniques de liaison, font de lui un bâtiment

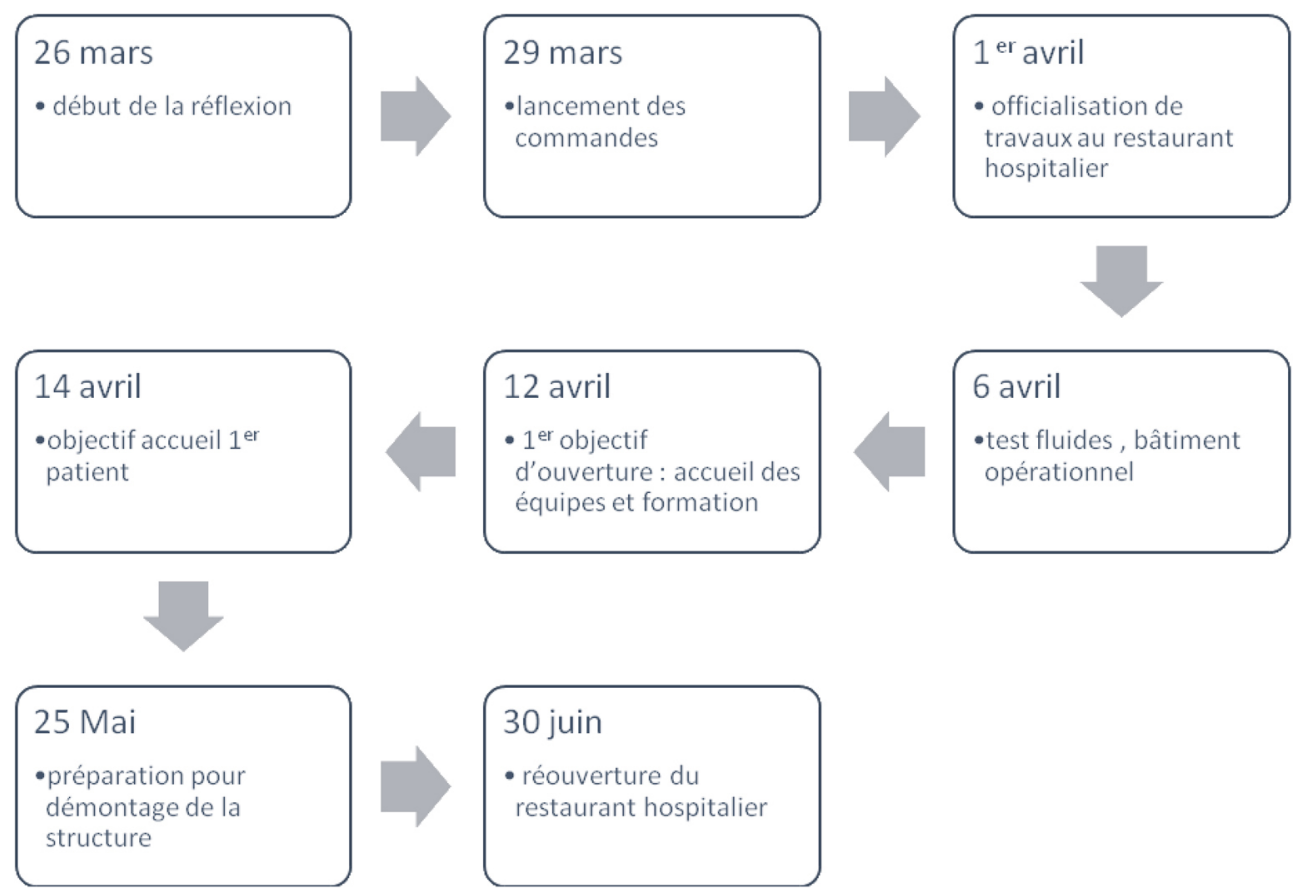

Fig. 1 Chronologie du projet Météor 
technique adapté à la situation. De plus, techniquement, il dispose d'une puissance électrique suffisante et d'un point de vue organisation spatiale intérieure de surfaces suffisantes permettant l'aménagement des différentes zones de soins et de supports envisagées. Le défi était de taille, car impliquant la transformation d'une salle sans aucune infrastructure biomédicale en réanimation éphémère, reconnue par le Conseil national professionnel d'anesthésie-réanimation médecine périopératoire comme le dernier choix à envisager [7].

\section{Organisation spatiale de Météor}

Météor a été imaginé avec un sas d'entrée comprenant trois lits et une zone administrative (Fig. 2). Cette zone était accessible de plain-pied par une rampe d'accès. Elle était d'une taille de $70 \mathrm{~m}^{2}$ et présentait en continuité immédiate un espace de $700 \mathrm{~m}^{2}$, doté de deux portes spécifiques permettant l'accès vers l'extérieur, séparé en trois zones par les couloirs techniques et qui comprenait 43 lits organisés selon un code couleur par plot de quatre lits. Un poste de soins doté de consoles de rappel des scopes était positionné à l'entrée de cette salle. À proximité, la zone bénéficiait d'une réserve et d'une salle de repos. Un couloir logistique dédié permettait l'accès spécifique à cette réserve ainsi que l'entrée des personnels venant prendre leur poste. Des chambres de garde étaient prévues l'étage.

\section{Conception technique}

La conception de cette nouvelle unité de 43 lits de soins critiques a été un véritable défi technique et technologique. Il s'agissait en partant d'une page vierge et d'un espace vide de toute activité de soins de trouver un moyen d'équiper rapidement des lits de réanimation. Ces lits impliquaient par essence une activité très pointue en termes d'infrastructures techniques, de dispositifs et d'équipements biomédicaux, mais également d'assurer une continuité de fonctionnement et de sûreté des installations en toutes circonstances. L'idée de conception était de définir un moyen de créer des installations techniques autour du lit (électricité, fluides médicaux, prises réseau) et de trouver des solutions pour pouvoir les alimenter depuis les organes de production. Mardi 30 mars 2021, lors d'une première réunion regroupant ingénieurs, techniciens et dessinateurs du groupe, la solution s'est imposé alors de créer des murs techniques démontables et réutilisables. Le concept était lancé. Ces murs techniques étaient composés de panneaux en bois stratifiés blancs. Ils présentaient les avantages de pouvoir être rapidement mis en

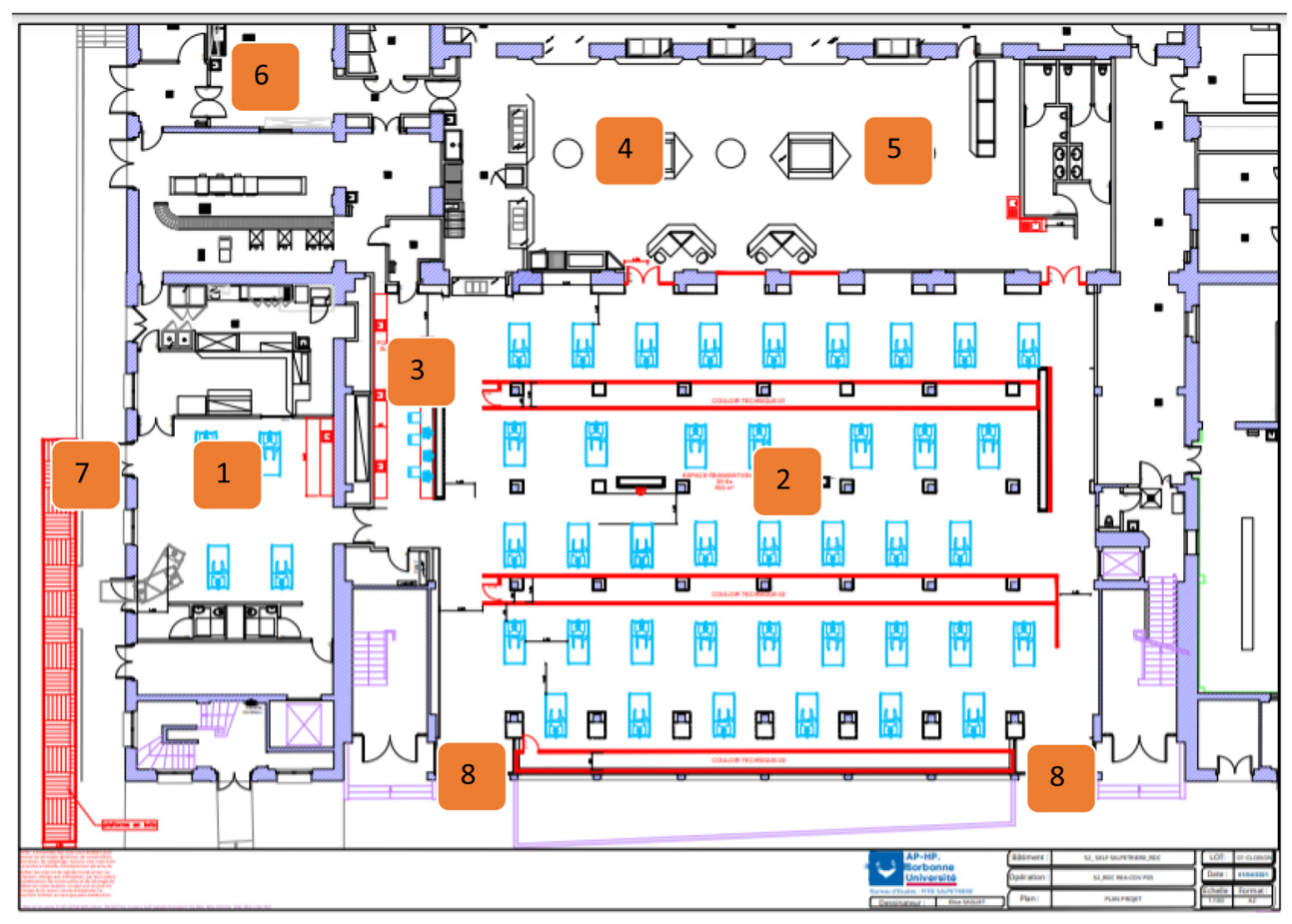

Fig. 2 Plan du projet Météor : 1 : zone d'accueil ; 2 : zone de soins ; $3:$ poste de soins ; $4:$ réserve ; $5:$ salle de repos ; $6:$ couloir logistique ; 7 : entrée ; 8 : sortie. Un patient est admis d'abord en zone d'accueil puis passe en zone de soins une fois le projet thérapeutique établi (source : Patrick Lhermitte, direction des investissements et des systèmes numériques, groupe hospitalier AP-HP-Sorbonne Université) 
œuvre, de permettre la constitution de couloirs techniques, tout en facilitant la circulation des lits. Cette conception rendait possible l'implantation des réseaux de câblage, de fluides, les protections électriques et en facilitait la distribution. Du point de vue de l'exploitation également, l'objectif de ces murs et couloirs techniques était de permettre d'intervenir à tout moment en cas d'incident, pour un dépannage ou un contrôle qualité préventif des installations, sans perturber ni interrompre l'activité de soins. Ces cloisons techniques ont été composées de plaques de dimensions unitaires 2,5 $\mathrm{m} \times 2,00 \mathrm{~m}$, sur lesquelles étaient fixées une gaine tête de lit et un bandeau électrique filant avec l'ensemble des prises électriques, informatiques et en fluides nécessaires à un lit de réanimation (Fig. 3). Un enjeu majeur de la conception de Météor a été la création intégrale d'un réseau de fluides médicaux d'une capacité suffisante pour desservir cette nouvelle entité. Il s'agissait de concevoir un réseau protégé, comprenant des armoires de détente avec toutes les panoplies que l'on positionnerait sur l'un des murs principaux du bâtiment, et de créer une centrale de vide dédiée, pour ne pas défavoriser le réseau existant. La définition du cahier des charges techniques est passée également, comme pour toute opération de restructuration hospitalière, par une définition des besoins des utilisateurs, en termes de circuits, de nombre et types de points d'eau, d'appareils d'éclairage, de report d'alarme, d'équipements biomédicaux, etc.

De manière générale, cette nouvelle entité a été conçue autour d'un principe fondamental qu'est la polyvalence. En effet, en cette période de crise sanitaire où le futur, même à court terme, est incertain, il était primordial d'anticiper au maximum en prévoyant des aménagements et équipements de haute technologie, adaptés aux attendus d'une unité de soins critiques pré- ou postréanimation, ainsi qu'aux besoins en termes de fonctionnement de service. Car même s'il

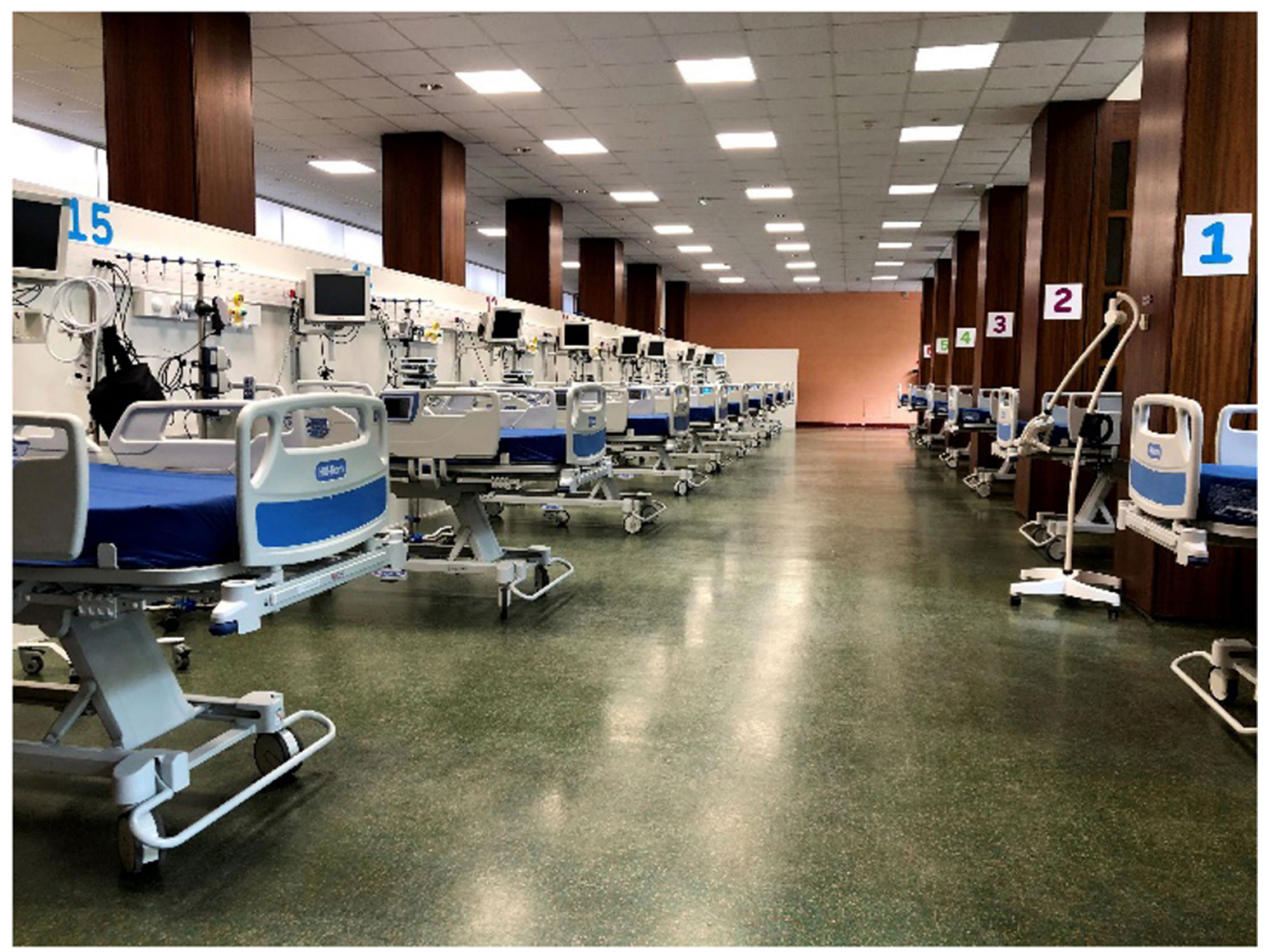

Fig. 3 Photo montrant la zone de soins Météor. Les cloisons techniques (blanches sur la photo) comprennent par lit : six prises de courant ; trois prises ondulées ; prises de fluides médicaux : deux d'oxygène, deux vides, deux d'air comprimé médical ; trois prises informatiques ; des prises de raccordement des équipements biomédicaux (source : Laurence Dentinger, direction de la communication, groupe hospitalier AP-HP-Sorbonne Université) 
s'agissait d'une unité créée pour répondre à un contexte d'urgence, il n'en restait pas moins que Météor se devait d'être conforme à toutes les normes et les attendus d'une activité aussi sensible, et aux pratiques institutionnelles pour garantir une qualité de soins et de prise en charge optimale. Dans cette optique, les plans techniques et d'aménagement ont donc ainsi été réalisés dans la journée du 30 mars. La direction du groupe hospitalier a donné son accord pour une fermeture de restaurant hospitalier à partir du $1^{\mathrm{er}}$ avril après le déjeuner en vue de démarrer la mise en œuvre in situ du projet. Pour pallier la baisse de $50 \%$ des activités de restauration, le second restaurant hospitalier existant sur le site permettait de prendre en charge une partie des besoins mais ne pouvait y répondre en totalité dans un contexte sanitaire où les gestes barrières devaient impérativement être respectés. Une des salles de garde de l'hôpital a été transformée en site de vente à emporter. Des barnums chauffés ont été installés dans les jardins pour permettre une restauration assise dans le respect des gestes barrières. Le déménagement du restaurant hospitalier s'est ensuite organisé avec l'objectif de protection des équipements afin de permettre par la suite une remise en état le plus rapidement possible en évitant toute détérioration anticipable.

\section{Réalisation}

Au-delà de sa conception particulièrement rapide, Météor a représenté de par sa mise en œuvre éclair un défi technique. Lancée le $1^{\mathrm{er}}$ avril 2021, avec la réalisation des alimentations primaires en fluides médicaux, électricité et fibre informatique, et pour tenir l'objectif d'une fin d'opération à l'issue du week-end de Pâques, le 6 avril 2021, sa réalisation a été effectuée dans son intégralité en cinq jours, fondée sur un roulement des équipes de deux fois huit heures journalières. Un planning a détaillé jour par jour le rôle de chacun. Les équipes du service technique, les techniciens biomédicaux et des informaticiens ont été sollicités pour leur expertise dans leur domaine de compétences. Un groupe de production de vide a été installé à proximité immédiate du bâtiment. Les premières gaines têtes de lit ont été façonnées prêtes à être montées sur les futurs murs techniques. Une cinquantaine d'ouvriers tous corps de métiers confondus (maçons, menuisiers, plombiers, électriciens, techniciens biomédicaux) ont été mobilisés. Dans un engagement collectif, de 7 à 23 heures, ils se sont activés, sans relâche, ainsi durant tout le weekend de Pâques, dans une parfaite coordination afin de rendre opérationnels les 43 lits promis. Ce délai de réalisation de cinq jours (intégrant aussi la réalisation des réceptions de conformité des fluides médicaux) a été d'autant plus remarquable, dans un contexte où les délais d'approvisionnement étaient de plus en plus restreints, conséquence directe de la crise sanitaire à travers le monde.

\section{Équipements}

L'ensemble des lits étaient équipés de manière standardisée avec un respirateur, un moniteur multiparamétrique, quatre pousse-seringues électriques. Météor était par ailleurs doté d'une machine à gazométrie artérielle délocalisée, d'une machine de diagnostic rapide pour la Covid, d'un portable de radiographie et d'un échographe. L'ensemble du matériel standard que l'on peut trouver dans une unité de soins critiques était à disposition : dispositif d'oxygénothérapie haut débit et de ventilation non invasive, respirateur de transport, ECG, fibroscope, échographe, matériel d'intubation difficile... Il n'était par contre pas prévu de recourir à des techniques de dialyse rénale ou hépatique ou encore à de l'assistance circulatoire. Dans le cas où un patient aurait nécessité un tel support, cela aurait impliqué son transfert vers une unité spécialisée. Une dotation en informatique a été effectuée pour permettre le raccordement de l'unité aux logiciels de soins. Concernant le financement des équipements, ils ont pour certain été achetés par anticipation, d'autres achetés de novo ou recyclés avec l'aide du service biomédical.

\section{Organisation de soins - Ressources humaines}

Que ce soit sur le plan médical ou paramédical, il avait d'emblée été anticipé que les effectifs destinés à travailler dans Météor n'avaient pas l'habitude de travailler ensemble, et pour un bon nombre pas l'habitude de travailler sur le site ni en soins critiques. Il a par ailleurs été anticipé que du fait des effectifs déjà en tension, les personnels de santé amenés à travailler sur Météor risquaient d'être en grande partie issus d'un turn-over important. Tout a donc été conçu pour faciliter leur exercice et permettre à chacun de s'adapter au plus vite à un environnement nouveau. L'ensemble des procédures médicales, paramédicales et administratives ont été écrites et au maximum standardisées (Annexes A, B). Dans cet esprit, des kits de soins ont été constitués pour les procédures spécifiques (par exemple pose de cathéter veineux central). Afin de faciliter la prise de repères par les personnels, les emplacements ont été repérés avec des codes couleur et un chariot de soins par groupe de quatre patients a été mis en œuvre. Le choix d'un ratio d'un infirmier et un aide-soignant pour quatre patients a été défini dans une recherche d'équilibre entre les ratios habituels de personnels en réanimation et le contexte de situation sanitaire exceptionnelle, avec la mise en avant de la supervision par un personnel expert. Le ratio en tenant compte de l'infirmier superviseur pour huit patients revenait ainsi à un infirmier pour deux patients et demi. Les ressources humaines en personnel médical et paramédical ont été définies en tenant compte du contexte impliquant des médecins superviseurs associés à des médecins 
moins expérimentés et de la même façon des infirmiers superviseurs associés à des infirmiers moins expérimentés en réanimation. Concernant les équipes médicales en journée, Météor prévoyait un médecin responsable de la zone d'accueil et un médecin responsable de la zone de soins à partir d'une ouverture à 16 patients. Pour la suite de la montée en charge, il était proposé d'avoir un binôme médecin senior et interne par groupe de huit patients (Tableau 1). Il était prévu la réallocation des effectifs en fonction de l'expertise des personnels et du flux réel des patients. Un groupe de quatre cadres de santé de proximité ayant contribué à l'organisation de Météor était prévu par relais dès l'ouverture afin d'assurer une présence permanente. La direction médicale aurait de même été présente autant que nécessaire en assurant de façon journalière des visites médicales. Une secrétaire chargée d'initier l'ensemble des démarches administratives et d'informatiser les dossiers à la sortie aurait été présente en journée. La présence d'une psychologue, d'un logisticien, d'un coursier, d'un agent technique, d'un agent de sécurité était aussi prévue. Compte tenu de la durée de séjour prévue comme courte, les équipes de kinésithérapeutes n'avaient initialement pas été sollicitées. Pour faciliter les flux, et en particulier les sorties des patients de la structure, il avait aussi été prévu que Météor s'appuie sur une cellule déjà existante au niveau du groupe hospitalier Sorbonne Université et en charge de l'aide au transfert de patients hospitalisés en soins critiques vers l'extérieur de l'AP-HP. Cette interaction aurait été construite en interface avec le directeur médical de crise AP-HP et le Samu zonal [8]. L'utilisation de l'informatique avait été conçue comme minimaliste de façon à simplifier la pratique de chacun en évitant les temps d'apprentissage spécifiques de chaque logiciel. Des dossiers, prescriptions et feuilles de surveillance papier ont été édités. L'organisation de l'ensemble des circuits a dû être anticipée et adaptée aux lieux, que ce soit pour les personnels, les patients et familles, la logistique, la pharmacie, les déchets ou l'approvisionnement hôtelier.

\section{Pharmacie}

Toujours dans un souci de simplification au quotidien et de rationalisation une fois les procédures standardisées, des dotations de médicaments, de solutés massifs et de dispositifs médicaux stériles ont dû être élaborées au regard de l'activité attendue en collaboration avec la pharmacie. Les tensions d'approvisionnement ainsi que les différentes formulations galéniques ont orienté sur les choix des spécialités retenues ainsi que sur les quantités. Une organisation plein-vide a été prévue pour faciliter les réapprovisionnements de l'unité. Ainsi, les armoires étaient préparées dans la pharmacie en échange des armoires vidées dans le service.

\section{Procédures équipes opérationnelles d'hygiène}

La visite des équipes opérationnelles d'hygiène a permis d'établir des règles de travail simple afin de protéger au mieux les personnels et les patients, tout en concevant des procédures faciles d'apprentissage, adaptées au lieu.

\begin{tabular}{|c|c|c|c|}
\hline & Organisation & Nombre & Équivalent temps plein \\
\hline \multirow[t]{2}{*}{ Médecin senior } & $\begin{array}{l}\text { Médecin zone de soins ( } 1 / 8 \text { lits, dont } \\
1 \text { superviseur) }\end{array}$ & 2 & 10 \\
\hline & Médecin zone d'accueil & 1 & \\
\hline \multirow[t]{2}{*}{ Interne } & Interne zone de soins ( $1 / 8$ lits) & 2 & 10 \\
\hline & Interne zone d'accueil & 1 & \\
\hline \multirow[t]{2}{*}{ Infirmière } & 1 pour 4 lits & 8 & 48 \\
\hline & 1 infirmier superviseur pour 8 lits & & \\
\hline Aide-soignant & 1 pour 4 lits & 6 & 36 \\
\hline Encadrement & 1 cadre expérimenté connaissant le site & 1 & 4 \\
\hline Autres catégories & 1 assistant médical administratif & 6 & 12 \\
\hline \multirow[t]{5}{*}{ de professionnels } & 1 psychologue & & \\
\hline & 1 logisticien & & \\
\hline & 1 coursier & & \\
\hline & 1 agent de sécurité & & \\
\hline & 1 technicien sur place & & \\
\hline
\end{tabular}




\section{Familles}

La structure de grande salle commune n'était pas une configuration optimale pour accueillir les familles de proches devant ne séjourner que peu de temps dans la structure. L'accès des proches a été considéré comme ne pouvant être que limité. Deux situations avaient été identifiées comme devant faire exception : la visite à un proche devant se faire intuber et la visite d'un proche en situation d'arrêt des thérapeutiques actives. Un accueil dédié par un des accès de la zone de soins principale avait donc été anticipé si nécessaire, avec la mise en paravents pour isoler le patient et sa famille. L'utilisation de tablettes pour faire de la visio avait aussi été envisagée.

\section{Communication autour du projet et enjeux}

La communication autour du projet Météor était un sujet critique avec des enjeux tant de communication interne qu'externe. La question était de savoir à quel moment il était possible de délivrer chaque information et quelle serait sa conséquence, que ce soit auprès du personnel ou des médias. En effet, bien que la volonté forte du projet était d'aller audelà de tout ce qui avait été déjà imaginé en termes de plans de crise, l'évocation de ce sujet pouvait tout aussi bien être le marqueur de l'idée que nous ne nous laisserions pas submerger mais aussi susciter l'incompréhension si le projet était mal expliqué. Le choix a été de dissocier la construction du projet et la communication laissant à la direction de la communication de l'AP-HP en accord avec les cellules de crise le choix de ce qui devait ou non être diffusé et selon quelles temporalités. Si des documents de travail interne ou des informations issues de réunions officielles (cellule de crise, réunion avec les organisations syndicales membres du comité d'hygiène et de sécurité au travail par exemple) ont circulé, peu d'éléments de communication officiels ont été diffusés en externe hormis un tweet du directeur général pour l'inauguration du lieu [9]. Une vidéo officielle, qui a été tournée par les services de communications internes afin de rendre compte de la construction et d'expliquer son objectif, n'a finalement pas été diffusée (Annexe C).

\section{Évolution du projet}

Le 12 avril (soit 15 jours après la prise de décision), l'unité était prête à ouvrir en accueillant en premier lieu les équipes constituées afin de valider les procédures écrites, les circuits et former les personnels à l'utilisation du matériel. Les premiers patients devaient être accueillis à partir du 14 avril, avec une ouverture progressive par plots de huit patients. L'unité n'a finalement pas accueilli de patient à cette date, l'ouverture de lits conventionnels supplémentaires dans les structures publiques et privées permettant de passer le pic qui est survenu dans les 15 jours suivants. La question du devenir de cette unité s'est alors posée, conduisant le projet médical à évoluer vers une unité de step down (unité de soins critiques prenant en charge des patients en voie d'amélioration). Le projet a alors été retravaillé en ce sens en adaptant les ratios de personnels, en ajoutant notamment la présence de kinésithérapeutes ou du matériel permettant la mobilisation et la verticalisation des patients. Cette grande salle commune potentiellement bruyante avec peu d'accès à la lumière naturelle ne rendait pas le lieu optimal pour cet usage, et l'unité n'a finalement pas ouvert non plus avec ce type de mission du fait d'une décrue dans l'épidémie. Au 25 mai, nous avons commencé à organiser le démontage de l'unité pour ensuite sécuriser et sauvegarder les installations restant sur place pour ensuite rénover le self et le rendre fin juin à sa première destination.

\section{Discussion}

Le projet a pu être mené avec efficacité dans un temps court. Cette efficience a été le fait d'une équipe de pilotage à la fois restreinte et multidisciplinaire ayant un objectif précis et carte blanche pour la mise en œuvre. Cette équipe projet s'est trouvée être dans les faits une cellule opérationnelle de mise en œuvre de décisions stratégiques prises en cellule de crise. Cette équipe projet a bien fonctionné parce qu'elle était à l'image de l'hôpital, c'est-à-dire multiprofessionnelle. Météor est un projet hospitalier au sein duquel l'ensemble des composantes médicales, paramédicales et techniques et de direction ont toutes été fondamentales et pour lequel chaque composante a pu donner son maximum en toute confiance. Météor est dans sa conception et son pilotage à l'image de nos cellules de crise au fonctionnement modernisé [10]. Météor a pu être imaginé et mis en œuvre du fait de l'excellence des équipes en place et des nombreuses unités éphémères construites en moins d'un an sur le site. La liste ne peut être exhaustive, mais on pourra citer par l'exemple la transformation du bâtiment ambulatoire en unité de postréanimation au cours de la première vague. Météor s'est construit en tant qu'unité de transition et non en tant qu'unité pérenne dans la mesure où les capacités de l'AP-HP paraissaient déjà avoir été étendues au-delà de limites raisonnables et que l'organisation d'un centre d'accueil avec des standards préservés avant transfert en extrarégional paraissait $\mathrm{du}$ fait des projections épidémiques une bonne solution. Des tentes pour agrandissement du service des urgences étaient déjà positionnées sur site. Le réaménagement de ces tentes pour faire face n'a pas été retenu parce qu'il ne permettait pas une extension aussi importante que l'aménagement d'un self. Un restaurant hospitalier présente par rapport 
à un gymnase comme cela a pu être fait ailleurs (France, Canada, Brésil, Espagne...) l'avantage essentiel d'être dans un hôpital et à proximité de l'ensemble des ressources nécessaires au fonctionnement d'une structure médicale de soins critiques. Le nombre de lits aménagé dans Météor est le fruit d'une projection dans le temps. Les travaux ne pouvaient être réalisés qu'une fois. Si nous choisissions de ne pas occuper totalement les lieux, refaire des travaux avec des patients sur site paraissait difficilement envisageable. À aucun moment la question financière n'a été un élément bloquant dans le pilotage de ce nouvel épisode de la crise Covid-19. Pourquoi aménager un self plutôt que réaménager un hôpital désaffecté comme cela a été fait dans d'autres régions ? La question a été débattue notamment du fait de l'existence sur Paris de lieu comme l'Hôtel-Dieu ou l'ancien hôpital militaire du Val-de-Grace. Cette solution n'a pas été retenue en raison notamment du fait qu'il n'est pas simple de réactiver des circuits logistiques, techniques et de pharmacie qui ont été délaissés. Par ailleurs, l'Hôtel-Dieu est devenu au cours de l'épidémie un site ambulatoire important tant pour le dépistage que pour la vaccination. Le maintien de son fonctionnement était donc complètement nécessaire à la lutte contre l'épidémie au niveau zonal. La construction d'un projet comme Météor est le fruit de capacités d'adaptation et de résilience, éléments indispensables pour faire à une situation sanitaire exceptionnelle. Les plans doivent exister, mais sont faits pour les adapter à la crise. Il fallait avoir confiance et donner confiance. Nous espérions ne finalement pas devoir faire rentrer de patient dans la structure tout en souhaitant si cela arrivait que les patients puissent être pris en charge le mieux possible malgré la crise par des équipes bien organisées. Créer Météor, c'était aussi donner une marge en plus dans une planification qui paraissait malgré les efforts déjà consentis arriver en bout de course. Le point le plus critique a au final été la question des ressources humaines. Les personnels ne peuvent se démultiplier, et la chronicisation de la crise nous rendait en cela vulnérables. La mise en route de Météor était possible avec les ressources humaines initialement de l'établissement sous réserve d'annuler les quelques jours de congé persistants, de faire appel à de l'intérim et aux réseaux de médecins ayant déjà travaillé sur la PitiéSalpêtrière. La pérennité du projet ne semblait pas pouvoir être assurée sans le renfort de nos confrères libéraux qui face à une épidémie potentiellement galopante auraient sans doute eu leurs activités plus que réduites du fait de la déprogrammation. L'hôpital public doit être au cœur de la société et d'un réseau de soins dépassant ses frontières géographiques. Il serait pertinent de se positionner sur l'avenir et de construire des mécanismes de renforts moins hypothétiques et plus stables en vue d'une future crise, telle une réserve sanitaire. Le caractère novateur du projet, dans une situation où compte tenu de la chronicisation de la crise la lassitude peut gagner des professionnels qui ne voient pas de sortie de crise, nous permettait d'envisager un sursaut supplémentaire dans la mobilisation de tous. Plusieurs personnels quoique déjà marqués par la crise en cours avaient émis le souhait de participer à ce nouveau défi, de même que des renforts hors $\mathrm{AP}-\mathrm{HP}$ intéressés à participer à cette innovation organisationnelle. Dans les éléments d'inquiétude, la capacité à faire sortir les patients vers des structures pérennes a été pointée comme un enjeu majeur. Dans ce cadre-là l'appui d'une cellule de régulation dédiée et du Samu zonal paraissaient essentiel [8]. Finalement, Météor aurait eu pour mission d'être tel un centre médical de tri et d'évacuation (CMTE) proposé par nos confrères du préhospitalier. Les cellules de crise et leurs directeurs médicaux de crise doivent à la fois innover dans le cadre hospitalier pour répondre à la crise mais aussi savoir s'inspirer d'outils déjà construits dans le cadre du préhospitalier. Cela passe par la formation et l'entrainement $[11,12]$. De taille plus petite et avec une intrication plus forte avec le Samu zonal, un autre CMTE avait été imaginé et mis en œuvre en parallèle au CHU Necker-Enfants-malades (AP-HP). En plus de cette collaboration forte avec le Samu, les critères de transfert élaborés précédemment par les sociétés savantes ont été retravaillés [13]. Il avait été ainsi acté par exemple en cellule de crise que les transferts en dehors de Météor ne dépendraient pas de l'accord des proches du fait du caractère particulièrement exceptionnel de la situation. Un autre point d'inquiétude résidait dans la crainte que la création de la structure génère un appel d'air non contrôlé de patients. Pour contrôler ce point, l'intégration au dispositif zonal était cruciale. Des règles d'entrée définies en commun et des objectifs clairs paraissaient indispensables à l'utilisation au mieux de cette structure sans glissement de fonctions non maîtrisé. À nouveau placer Météor au sein du dispositif zonal était en ce sens totalement indispensable. La transformation d'un restaurant hospitalier en réanimation fonctionnelle et équipée prend, quand bien même elle est issue d'un processus accéléré tel que dans le cas de Météor, un temps incompressible. Elle ne peut donc être une réponse à un évènement aigu, mais elle est une solution à un événement à cinétique lente en termes de recours aux soins critiques. Sur le site, d'autres solutions qui prennent moins de temps ont été mises en œuvre. Des chambres ont par exemple été doublées (deux patients par chambre) dans une réanimation chirurgicale polyvalente. Sous réserve de chambre de tailles suffisantes et d'une anticipation technique (ajout de prise d'oxygène, ajout de prise d'air comprimé médical, de vide, de prises électriques et de postes informatiques dédiés) et d'une réflexion sur l'organisation des soins pour éviter toute erreur liée à la suroccupation inhabituelle des chambres, cette solution présente l'avantage de pouvoir être mise en œuvre rapidement et de permettre de faire face à un événement nécessitant une augmentation rapide en nombre de lits de soins critiques. Dans le cadre de la nécessité d'une augmentation 
rapide en lits de soins critiques, il ne faut bien sûr pas oublier nos salles de surveillance postinterventionnelle [14]. Audelà, un appui exceptionnel peut venir de l'extérieur, comme nous l'a montré l'exemple du service de santé des armées à Mulhouse en déployant un élément mobile de réanimation adapté au contexte Covid [15]. Le choix de mettre en œuvre une solution plutôt qu'une autre ne peut être piloté au niveau d'un service, mais doit être pilotée en cellule de crise chaque solution présentant des avantages et des inconvénients. Météor a un coût et comprend des risques à mettre en face des enjeux de la situation où le projet a été initié. Il n'était de toute façon pas envisageable de laisser des patients sans solution de prise en charge. Météor doit être considéré comme un outil supplémentaire et transitoire face à une crise supposée aller au-delà de toutes les prévisions. Les hôpitaux civils de Lyon à la recherche de nouvelles options d'adaptation face à la crise ont échangé avec les équipes de l'AP-HP au printemps 2021 afin de partir de l'expérience acquise. Penser la postcrise, c'est tirer des leçons des évènements actuels pour s'adapter dans le futur. Il parait ainsi nécessaire d'anticiper sur les7 prochaines constructions ou rénovations hospitalières et penser des lieux modulables qui, au quotidien, ont une fonction spécifique mais qui pourraient être adaptés, en plus des lieux déjà dédiés au soin. Du fait de cette polyvalence, ils pourraient par exemple être d'emblée câblés et les procédures de transformations avec leur délai de mises en œuvre décrites au préalable. Il parait pertinent que nos CHU s'ils n'ont pas déjà imaginé en leurs murs ce genre de lieux se projettent dès maintenant. Nous avons décrit la transformation d'un restaurant, d'autres ont imaginé par exemple la transformation de parking en Israël [16]. Cette adaptation au temps du Covid est partie de plans préexistants pour pouvoir faire face aux conflits armés [17]. Les Suisses quant à eux ont construit pour faire face au temps de guerre des hôpitaux souterrains. Leur utilisation pour la Covid a été discutée. Néanmoins, le manque d'entretien a freiné la mise en œuvre. Plus d'un an de Covid a montré la nécessité de savoir transformer l'hôpital et augmenter les capacités en soins critiques. Météor comme d'autres projets ont montré la particulière difficulté de s'adapter à augmenter rapidement la ressource en personnels compétents. Il faut penser l'avenir et anticiper la prochaine crise. Il s'agit d'avoir au côté des personnels qui travaillent au quotidien en soins critiques un volant de personnels supplémentaires pouvant suivre un parcours défini de formation et de recyclage, voire de séjours d'immersion et volontaires pour si besoin venir renforcer les soins critiques. Ainsi, une réserve sanitaire de réanimation a commencé à voir le jour à l'issue de la première vague et doit être pérennisée et renforcée [18].

\section{Conclusion}

Le projet Météor a su fédérer l'ensemble des équipes de la communauté hospitalière, qu'il s'agisse des directions, des fonctions techniques, logistiques, médicales et paramédicales, autour d'un projet ambitieux et unique. Météor a représenté un défi technologique et technique, d'autant plus renforcé dans un contexte de crise, complexifiant depuis plusieurs mois les délais de fabrication et d'approvisionnement des matières premières. Météor révèle parfaitement bien les ressources et compétences présentes au sein des équipes du Groupe hospitalier, mais également l'implication, la motivation et l'engagement sans faille de celles-ci. Transformer un self en réanimation n'est ni irréaliste ni impossible. Il faut penser l'avenir pour que l'hôpital garde cette capacité à penser autrement et sortir si nécessaire du cadre en cas de situation sanitaire exceptionnelle.

Liens d'intérêts : M. Borel déclare un conflit d'intérêts avec la société Medusims (conseiller pédagogique) et avec la société Emergensim (formateur). P. Lhermite, N. Fleury, M.-A. Ruder, C. Fazilleau, E. Boudon, T. Lemarec, J. Mayaux, J.-M. Constantin et M. Raux déclarent ne pas avoir de liens d'intérêts.

\author{
Éléments remarquables \\ - Cellule stratégique de pilotage \\ - Cellule opérationnelle multiprofessionnelle \\ - Adaptation et résilience \\ - Initiative locale mais intégration zonale
}

\section{Pistes d'amélioration}

- Anticipation

- Réserve sanitaire de réanimation

- Intégration dans les futurs aménagements hospitaliers

- Intégration dans les plans de crise 


\section{Références}

1. Cauchemez S (2021) Projection à court terme des besoins hospitaliers pour les patients Covid-19. https://modelisationcovid19.pasteur.fr/realtime-analysis/hospital/ (Dernier accès le 9 juin 2021)

2. Lagadec P (2015) Journal de bord des temps chaotiques. Le continent des imprévus. Ed Manitoba. Les Belles Lettres, Paris

3. Helsloot I, Boin A, Jacobs B, et al (2012) Mega-crises: understanding the prospects, nature, characteristics and effects of cataclysmic events. Ed Charles Thomas Publisher, Springfield

4. Ghorbanian A, Bahadori M, Nejati M (2012) The relationship between manager's leadership styles and emergency medical technicians' job satisfaction. Australas Med J 5:1-7

5. Lamhaut L, Nivet CM, Dagron C, et al (2020) Retour d'expérience des évacuations par train à grande vitesse de patients en syndrome de détresse respiratoire aiguë sur infection à Covid19 : les missions Chardon. Ann Fr Med Urgence 10:288-97

6. Lecarpentier E, Travers N, Jbelli C, et al (2020) Retour d'expérience sur le centre médical de coordination et d'évacuation aérien Covid - Paris-Orly. Ann Fr Med Urgence 10:278-87

7. Conseil national professionnel d'anesthésie-réanimation médecine périopératoire (2020) Guide d'aide à la mise en place et à la gestion d'une réanimation éphémère. https://sfar.org/guidedaide-a-la-mise-en-place-et-a-la-gestion-dune-reanimation-ephemere/ (Dernier accès le 24 mai 2021)

8. Langlois M, Borel M, Clovet O, et al (2020) Cellule de coordination des flux sortants des réanimations en période Covid-19. Ann Fr Med Urgence 10:327-32

9. Hirsch M (2021) Inauguration de Météor. https://twitter.com/MartinHirsch/status/1383060520488554506 (Dernier accès le 9 juin 2021)

10. Ministère des Solidarités et de la Santé (2019) Guide d'aide à la préparation et à la gestion des tensions hospitalières et des situations sanitaires exceptionnelles. https://solidarites-sante.gouv.fr/
IMG/pdf/guide_situation_sanitaire_exceptionnelle.pdf (Dernier accès le 24 mai 2021)

11. Médecine Sorbonne Université (2021) Le directeur médical de crise. https://dpc.sorbonne-universite.fr/offre-de-formation/formations-pour-les-medecins/le-directeur-medical-de-crise-dmcmodule-1-de-la-theorie-a-la-pratique/ (Dernier accès 23 mai 2021)

12. Borel M, Damm C, Debien B, et al (2019) S'exercer à l'afflux massif de victimes hospitalières... Comment faire ? Ann Fr Med Urgence 9:163-72

13. Raux M, Gayat E (2020) Critères de transférabilité interrégionale d'un patient de soins critiques d'un établissement de santé à un autre. https://www.sfmu.org/upload/consensus/rpc_list tranfert_SFAR_SRLF_SFMU201217.pdfhttps://www.sfmu.org/ upload/consensus/rpc_list_tranfert_SFAR_SRLF_SFMU201217. pdf (Dernier accès le 24 mai 2021)

14. Clavier T, Bruckert V, Abraham P, et al (2021) The use of postanaesthesia care units as a supply of ICU beds while maintaining scheduled surgery: a cross-sectional web-based feasibility survey in France. J Clin Anesth 71:110244

15. Kuteifan K, Pasquier P, Meyer C, et al (2020) The outbreak of Covid-19 in Mulhouse hospital crisis management and deployment of military hospital during the outbreak of Covid-19 in Mulhouse, France. Ann Intensive Care 10:59

16. Leshem E, Klein Y, Haviv Y, et al (2020) Enhancing intensive care capacity: Covid-19 experience from a tertiary center in Israel. Intensive Care Med. 25:1-2

17. Halberthal M, Berger G, Hussein K, et al (2020) Israeli underground hospital conversion for treating Covid-19 patients. Am J Disaster 15:159-67

18. Conseil national professionnel d'anesthésie-réanimation médecine périopératoire (2021) 2021-2030. L'avenir des soins critiques. Quels soins critiques entre deux crises. https://sfar.org/ livre-blanc-lavenir-des-soins-critiques-2021-2030/?utm_source $=$ rss\&utm_medium $=$ rss\&utm_campaign=livre-blanc-lavenirdes-soins-critiques-2021-2030 (Dernier accès le 5 juillet 2021) 


\section{Annexe A. Exemple de procédure standardisée}

\begin{tabular}{|l|l|l|}
\hline Procédure & Unité METEOR & $05 / 04 / 2021$ \\
\hline Objet: Prescriptions standardisées & \\
\hline
\end{tabular}

1. CONSIGNESIDE:

- Surveillance/3h avec EtCO2, objectifs (SpO2 90-93\%, PAM) $>55 \mathrm{mmHg}$, RASS et BPS si phase de réveil)

- aspiration système clos,

- isolement respiratoire + gouttelettes,

- pas de contention si curarisation,

- à jeun si $O 2>6 \mathrm{~L} / \mathrm{min}$

2. DISPOSITIFSINVASIFS:

- KTA/KTC/SNG/SU + Préciser position

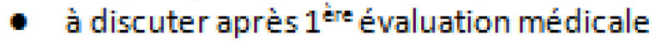

3. BILANS STANDARDS:

- ENTREE : GDS, ionogramme sanguin, BHC, NFS, TP/TCA, PCT/CRP, troponine, DDimères, ionogramme U, RXT (+/-après IOT+ SNG + KTC), Echographie pulmonaire/ETT, ECG

- QUOTIDIEN : Bilan complet, Triglycéride/48h si propofol, GDS pluriquotidiens

\section{INFECTIEUX:}

- ENTREE : recherche COVID si non fait (privilégier prélèvement profond si IOT, prévoir $2 \mathrm{kme}$ prélèvement si négatif), aspiration trachéale que si arguments pour surinfection, antigénuries légionnelle et pneumocoque si non fait

5. VENTILATION :

02, Sonde IOT/VAC/NOSTEN (humidificateur chauffant à discuter)

\section{MEDICAMENTS :}

- Perfusion de base IV (restriction hydrique, à adapter aux données de la clinique)

- Sédations: propofol IVSE + sufentanil

- Curares : bésilate de cisatracurium20 $\mathrm{mg} / \mathrm{h}$ (tenter arrêt quotidien si possible)

- Penser à prescrire les traitements adjuvants : DV.

- Esoméprazole si indication (noradrénaline, corticothérapie, ATCD UGD...)

- Protocole insuline

- Traitement habituel si possible

- Antibiothérapie uniquement si arguments pour surinfection bactérienne

- Dexaméthasone $6 \mathrm{mg} / \mathrm{j}$ pendant 10 jours $+/$ - ivermectine ( $11 / 17,200 \mu \mathrm{g} / \mathrm{kg}$ en 1 prise)

- Prophylaxie anti thrombotique majorée (malades pro-coagulants ++, enoxaparine sodique0.4x $2 /\rfloor$ si Cl>15, ac contrôle antiXa entre $3 \mathrm{~km}$ et $4 \mathrm{imm}$ dose) Interrompre tout traitement hormonal ou apparenté 


\section{Annexe B. Exemple de kit constitué}

\section{Kit intubation}

1.

2.

3.

4.

5.

6.

7.

8.

9.

10.
1 vidéolaryngoscope

2 lames de vidéolaryngoscope $n^{\text {os }} 3$ et 4

Sondes d'intubation 7/7.5/8/8.5

1 laryngoscope à lumière froide

2 lames $n^{\text {os }} 3$ et $^{\circ} 4$

1 canule de Guedel $n^{\circ} 2$

1 seringue de $20 \mathrm{ml}$

1 rouleau de sparadrap

Clamp

Masque FFP2 + lunettes
Annexe C. Rationnel de Météor et mise en ouvre (Crédit vidéo : Cassandre Breysse, direction de la communication, AP-HP)

https://www.youtube.com/watch?v=6UEs_WEJrPU\&ab_channe l=AP-HP (Matériel supplémentaire) 